\title{
RESULTADOS DEL TRATAMIENTO CON METOTREXATE LOCAL BAJO GUÍA ECOGRÁFICA EN PACIENTES CON EMBARAZO ECTÓPICO NO COMPLICADO CON INDICACIÓN QUIRÚRGICA, EN EL SERVICIO DE GINECOLOGÍA DEL INSTITUTO NACIONAL MATERNO PERINATAL
}

\author{
Marco Antonio Garnique Moncada', Luis Alberto Carpio Guzmán², Humberto Adler Izaguirre Lucano³, \\ Agustín Murga López ${ }^{4}$
}

\begin{abstract}
RESUMEN
Objetivo. Evaluar los resultados de una serie de casos consecutivos de embarazos ectópicos no complicados con indicación quirúrgica de acuerdo a nuestras guías actuales de manejo del embarazo ectópico en el Institutito Nacional Materno Perinatal (INMP) que fueron manejadas con inyección local de metotrexate (MTX) guiado por ecografía transvaginal, además de la aplicación del mismo por vía intramuscular. Materiales y métodos. Es un estudio prospectivo, longitudinal, de intervención. Se estudiaron nueve pacientes con diagnóstico de embarazo ectópico $(\mathrm{EE})$ no complicado con indicación quirúrgica en el servicio de hospitalización de ginecología en el INMP, a quienes se les ofreció tratamiento mediante la administración de MTX local bajo guía ecográfica e intramuscular y realizar el seguimiento tanto ecográfico como de la hormona gonadotrofina coriónica fracción beta (hCG Beta) sérica hasta la resolución completa del embarazo ectópico. Se estimaron las frecuencias absolutas y relativas para las variables categóricas y medidas de tendencia central y de dispersión para las variables continuas. Resultados. El tratamiento combinado con MTX local y MTX intramuscular fue exitoso en ocho de las nueve pacientes $(88.8 \%)$. El nivel sérico de hCG Beta promedio con la que se inició el tratamiento fue $9499 \mathrm{UI} / \mathrm{L}$. El tiempo promedio del procedimiento en sala de operaciones fue 12.6 minutos. Una paciente tuvo que ser intervenida por laparoscopía por embarazo ectópico roto. Conclusiones. El tratamiento del EE no complicado con indicación quirúrgica mediante tratamiento combinado con MTX local e intramuscular parece ser seguro y efectivo. El tratamiento indicado podría ser de primera línea en pacientes que deseen preservar la fertilidad y parece ser costo efectivo ya que es mínimamente invasivo.
\end{abstract}

Palabras clave: Embarazo ectópico no complicado con indicación quirúrgica; administración de metrotexate local ecoguiado (Fuente: DeCS BIREME)

\section{RESULTS OF TREATMENT WITH METHOTREXATE UNDER LOCAL GUIDE ULTRASOUND IN PATIENTS WITH PREGNANCY COMPLICATED ECTOPIC SURGICAL INDICATION IN THE GYNECOLOGY SERVICE OF THE NATIONAL MATERNAL PERINATAL INSTITUTE}

\begin{abstract}
Objective. Evaluate the results of a series of consecutive cases of ectopic pregnancies complicated with surgical indication according to our current management guidelines of ectopic pregnancy in the National Maternal Perinatal Institute (INMP) who were managed with local injection of methotrexate (MTX) guided by transvaginal ultrasound, in addition to its application intramuscularly. Materials and methods. It is a prospective, longitudinal intervention study. Nine patients with diagnosis of ectopic pregnancy (EP) uncomplicated with surgical indication in the hospital department of gynecology at INMP, who were offered treatment by administering local MTX under ultrasound and intramuscular guide and track were studied both ultrasound and serum chorionic gonadotropin beta fraction (hCG beta) until complete resolution of ectopic pregnancy. Absolute and relative frequencies for categorical variables and measures of central tendency and dispersion for continuous variables were estimated. Results. Combined treatment with MTX and Local intramuscular MTX was successful in eight of nine patients $(88,8 \%)$. The average serum level of hCG Beta with which treatment was started was 9499 IU / L. The average procedure time in the operating room was 12,6 minutes. One patient had to be operated by laparoscopy for ruptured ectopic pregnancy. Conclusions. EE treating uncomplicated with surgical indication by combined treatment with local and intramuscular MTX appears to be safe and effective. The treatment may be indicated frontline in patients wishing to preserve fertility and appears to be cost effective as it is minimally invasive.
\end{abstract}

Key words: Ectopic pregnancy uncomplicated with surgical indication; ultrasound-guided local methotrexate administration (Source: MeSH NLM).

\footnotetext{
Médico Gineco Obstetra Asistente. Servicio de Medicina Reproductiva. Instituto Nacional Materno Perinatal . Lima-Perú.

Médico Gineco Obstetra . Jefe del Servicio de Ginecología Especializada. Instituto Nacional Materno Perinatal . Lima-Perú.

Médico Gineco Obstetra . Jefe del Servicio de Ginecología Patológica. Instituto Nacional Materno Perinatal . Lima-Perú.

Médico Gineco Obstetra . Jefe del Departamento de Ginecología. Instituto Nacional Materno Perinatal . Lima-Perú.
} 


\section{INTRODUCCIÓN}

El embarazo ectópico (EE) está definido como la implantación y desarrollo del ovocito fertilizado fuera de la cavidad uterina ${ }^{1}$. EI EE ocurre en $1,5-2,0 \%$ de todos los embarazos, ésta es una de las patologías obstétricas que aunque su incidencia ha aumentado, la mortalidad ha disminuido, debido al diagnóstico y tratamiento antes de la ruptura del $\mathrm{EE}^{2}$.

El EE es predominantemente tubárico (97\%), aunque puede también implantarse en el cérvix, ovario, abdomen. Un factor común relacionado es la alteración o patología en la trompa uterina en las que se incluyen cirugía tubárica, infecciones del tracto genital a la cabeza la enfermedad pélvica inflamatoria, EE previo y útero expuesto al dietilestilbestrol 3; otros factores incluyen pacientes con diagnóstico de infertilidad uso de tratamientos de reproducción asistida, cirugía pélvica o abdominal previa y el consumo de tabaco ${ }^{4}$.

La mortalidad materna subsecuente de un EE es poco común en países desarrollados. En el Reino Unido del 2003 al 2005, se reportaron 10 muertes maternas por EE (0,47 por cada 100,000 embarazos). La morbilidad materna está limitada y relacionada a dolor, requerimiento de transfusión y complicaciones quirúrgicas ${ }^{5}$.

El tratamiento a nivel mundial ha progresado desde ser primariamente quirúrgico por laparotomía a ser cada vez mínimamente invasivo o manejar médicamente con $\mathrm{MTX}^{6,7}$

En la actualidad están reportados diversas formas de manejo dependiendo de la forma clínica del EE, así como de la experiencia del ginecólogo; es posible que todos estén de acuerdo en realizar una laparotomía de emergencia si la paciente llega con shock hipovolémico por hemoperitoneo masivo causado por embarazo ectópico roto, pero con la mejora de métodos diagnósticos no invasivos, como los test de sensibilidad tanto urinarios como sanguíneos, así como la ecografía transvaginal de alta resolución ha hecho posible el diagnóstico precoz de $E E^{8}$. Como consecuencia la presentación clínica del EE ha cambiado desde comprometer la vida y necesitar de una cirugía de emergencia a una condición benigna frecuentemente asintomática con opciones de tratamiento diferentes al quirúrgico ${ }^{9}$.

Los protocolos de manejo para EE están basados en una serie de parámetros como tamaño de la masa anexial, presencia o no de embrión, presencia o no de latidos cardioembrionarios, nivel de la sub unidad beta, etc. que revisados mediante una serie de análisis estadísticos valoran el coste efectividad de uno u otro tratamiento. De esta manera se opta por manejo expectante; manejo médico o manejo quirúrgico.

El manejo expectante se da en mujeres en quienes se sospecha de embarazo ectópico y bajos niveles séricos de hCG, se monitoriza mediante ecografía transvaginal y niveles séricos de hCG el desarrollo del embarazo. Una inicial presentación del EE puede ser sospechado mediante ecografía transvaginal por un anillo ectópico o masa anexial sin líquido libre. Si estos hallazgos ecográficos están ausentes se puede hacer el diagnóstico tentativo de Gestación de Localización Indeterminada (GLI), siempre y cuando los niveles séricos de B-hCG estén debajo de la zona discriminatoria de 1,000 a 1,500 UI/L. La evaluación en el tiempo aclarará el desarrollo de un embarazo intrauterino (34\%) o el de un EE (11\%), el $55 \%$ se resolverá espontáneamente 5,10 .

El manejo médico con MTX es para mujeres con embarazo ectópico visible y bajas concentraciones séricas de hCG o persistencia de GLI ${ }^{11}$. Sin embargo puede el embarazo ectópico seguir un curso natural o limitarse asimismo, resultando en un aborto tubárico o reabsorberse. En algunas cohortes de estudios esperan evaluando permanentemente y tratando sólo los casos seleccionados de embarazos ectópicos que siguen su curso ${ }^{12}$.

Al momento en el Perú, cuando esta alternativa no da resultado o cuando existen criterios para tratamiento quirúrgico siendo no complicado (Beta hCG > $5000 \mathrm{UI} / \mathrm{L}$ o embrión con actividad cardiaca) la alternativa es una cirugía idealmente por vía laparoscópica.

El manejo Quirúrgico de embarazos ectópicos no complicados con indicación quirúrgica puede ser tanto a cielo abierto (laparotomía) o por vía laparoscópica, dependiendo de la experiencia del ginecólogo y de la infraestructura hospitalaria y nos referiremos a pacientes con embarazo tubárico que representa el $97 \%$ de los embarazos ectópicos. En este tipo de intervenciones se puede optar por la realización de una cirugía radical (salpinguectomía) o conservadora (salpingostomía o salpingotomía).

La laparoscopía quirúrgica es mejor que la laparotomía, por sus resultados en relación a menor sangrado, corta estancia hospitalaria, menor tiempo quirúrgico, menos analgesia y corto tiempo de convalecencia, sin embargo en las áreas rurales la laparotomía es todavía la cirugía standard ${ }^{13}$

La revisión de Cochrane reportó que la salpingotomía laparoscópica es menos exitosa que la salpingotomía por laparotomía en la erradicación del embarazo tubárico (RR 0,$90 ; 95 \% \mathrm{Cl} 0,83-0,97$ ). Esto fue debido a la alta persistencia de tejido trofoblástico en el grupo de cirugía laparoscópica (RR 3,6,95\% Cl 0,63-21). Sin embrago no hubo diferencia en la concepción futura (RR 1.2, 95\% $\mathrm{Cl}$ 0,88-1,5) o de embarazo ectópico de repetición (RR $0,43,95 \% \mathrm{Cl} 0,15-1,2)$ entre el grupo de laparoscopía y el grupo de laparotomía ${ }^{14}$.

El Real Colegio de Ginecología y Obstetricia recomienda que la salpingotomía podría ser considerada como tratamiento primario en la presencia de trompa normal 
o daño tubárico contralateral a menos que sea evidente un hidrosalpinx o daño severo en la trompa ipsilateral ${ }^{15}$. De otra forma una salpingotomía puede comprometer el éxito de una FIV ${ }^{16}$.

Un problema con la salpinguectomía es el deterioro en el suministro de flujo sanguíneo al ovario ya que ésta disminuye la reserva ovárica ${ }^{17,18}$. El otro problema con el tratamiento quirúrgico conservador es la persistencia del trofoblasto. Ésto es extremadamente raro en quienes han tenido una salpinguectomía, pero puede ocurrir hasta en el $8 \%$ de mujeres sometidas salpingotomía. Hence, hizo un importante seguimiento a pacientes sometidas a salpingotomía con medidas de B-hCG y trató efectivamente con MTX en dosis única de $50 \mathrm{mg} / \mathrm{m} 2$ o 1 $\mathrm{mg} / \mathrm{Kg}$, si permanecían elevadas.

Se ha reportado manejo con MTX local, primero por vía laparoscópica y luego guiado por ecografía con éxito de hasta el $100 \%$ aunque con pocos casos y en EE intersticiales como los reportados por Hafner y col ${ }^{19}$. Así mismos se ha reportado el uso de diversos citotóxicos diferentes al MTX, como CLK, Actinomicina D, glucosa híper osmolar, Etopósido con diferentes porcentajes de éxito aunque siempre con pocos casos.

Con el mejoramiento de los equipos de ecografía de alta resolución así como dispositivos para la inyección local del MTX que podría ser más efectivo que aplicarlo por vía sistémica para embarazos más prolongados en los que el MTX solo intramuscular reporta una tasa de falla de hasta el $30 \%$, la aplicación local de citotóxicos se ha reportado como exitoso aunque las mayoría de publicaciones aún son muy limitadas y en general reporte de $\operatorname{casos}^{20-23}$.

No se han encontrado estudios, ni reporte de casos publicados en el Perú en relación a tratamientos de embarazos ectópicos con citotóxicos locales.

El Objetivo del siguiente estudio fue evaluar los resultados de 9 pacientes con diagnóstico de embarazo ectópico no complicado con indicación quirúrgica a quienes se les ofreció un tratamiento con citotóxico (MTX o CIK) local guiado por ecografía además de la administración de MTX por vía intramuscular.

\section{MATERIALES Y MÉTODOS}

El estudio fue prospectivo, longitudinal, de intervención, llevado a cabo entre el $1^{\circ}$ de Octubre del 2013 al 30 de Abril del 2014, en el Servicio de Ginecología del INMP. Lima-Perú.

Se estudiaron nueve pacientes con diagnóstico de EE no complicado con indicación quirúrgica en el servicio de hospitalización de ginecología del INMP, a quienes se les ofreció tratamiento mediante la administración de Citotóxico (MTX o CLK) local bajo guía ecográfica e intramuscular de MTX y realizar el seguimiento tanto ecográfico como del $\beta$-HCG sérico hasta la resolución completa del embarazo ectópico.

Los criterios de inclusión fueron pacientes hemodinámicamente estables, con evaluación ecográfica por el servicio de ginecología compatible con EE no roto, examen de $\beta-H C G$ > $5000 \mathrm{UI} / \mathrm{L}$, EE con embrión activo $O E E$ que no respondió a tratamiento médico con MTX manejada por el servicio, evaluación de perfil de coagulación, hepático y renal dentro de parámetros normales, pacientes con deseo de fertilidad futura y firma del consentimiento informado.

Todas las pacientes fueron evaluadas con Ecografía del servicio de ginecología corroborándose el diagnóstico de embarazo ectópico no complicado. La evaluación se realizó con Ecógrafo Aloka prosound Modelo SSD$3500 S V$. Todas las pacientes tuvieron examen de $\beta-\mathrm{HCG}$ actual al momento del tratamiento.

Se intervinieron dos grupos de pacientes, aquellas con diagnóstico de embarazo ectópico no complicado con indicación quirúrgica (7 pacientes) y aquellas que siendo manejadas con tratamiento médico con metrotexate los niveles de $\beta$-HCG no disminuían o estaban en aumento (2 pacientes).

A todas las pacientes intervenidas independientemente del grupo se les aplicó una dosis de MTX intramuscular a razón de $50 \mathrm{mg} / \mathrm{m}^{2}$ de superficie antes de ser intervenidas. El procedimiento de administración local del MTX se realizó en sala de operaciones del Centro Quirúrgico del INMP. Utilizándose una aguja de aspiración folicular 17G de doble lumen, un Ecógrafo Alokaprosound 2 Modelo RMT-PS2 con transductor transvaginal al cual se le acopló una guía metálica para la aguja de aspiración, y administración local de MTX 50mg.

La paciente en ayunas pasaba a sala de operaciones donde el anestesiólogo procedía a la anestesia, se colocaba en posición de litotomía, y se procedía a realizar la asepsia antisepsia perineo vaginal, se procedía a examinar la masa anexial por ecografía mediante el transductor transvaginal con funda aséptica para el transductor y acoplada ya la guía metálica para aguja de aspiración, una vez identificado los hallazgos se procedía a punzar el saco gestacional aspirándose el líquido intrasacular y acto seguido se le administraba el MTX a dosis de $50 \mathrm{mg}$ por el lumen diferente al de aspiración. Al notar el colapso del saco gestacional evaluada por ecografía en tiempo real y la visualización del líquido citrino a la aspiración del saco se corroboraba la ubicación adecuada de la aguja y por lo tanto se iniciaba con la inyección del MTX, evidenciándose la nueva distención del saco y visualización del MTX administrado. Culminado el procedimiento se retiraba la aguja sin dejar de hacer compresión en fondo de saco con el transductor por unos cinco minutos tratando de 
hacer hemostasia por compresión sabiendo que la aguja estaba atravesando partes blandas y la trompa uterina.

Todas las pacientes estuvieron bajo evaluación post procedimiento por dos horas en sala de recuperación de centro quirúrgico y enviadas al servicio de hospitalización si se encontraban hemodinámicamente estables. Todas las pacientes fueron evaluadas a las veinticuatro horas post procedimiento mediante ecografía transvaginal en el servicio de ginecología para descartar líquido libre de regular cuantía. Se les tomó examen de $\beta-H C G$ al cuarto día y se les dio alta con $\beta-H C G$ en disminución y/o ecografía transvaginal sin alteraciones en relación a la del control de veinticuatro horas tomada post procedimiento. El control fue realizado con indicación a las pacientes de acudir semanalmente por consultorios externos en la unidad de infertilidad del INMP con $\beta$-HCG y ecografía transvaginal.

\section{RESULTADOS}

Entre el $1^{\circ}$ de Octubre del 2013 al 30 de Abril del 2014 se Hospitalizaron en el servicio de ginecología 698 pacientes; de las cuales $84(12,03 \%)$ ingresaron por embarazo ectópico. De éstas $51(60,71 \%)$ tuvo manejo quirúrgico y casi todas por el servicio de emergencia, por lo tanto ingresaron como post operadas, salvo una paciente que la intervino el ginecólogo del servicio de hospitalización de forma electiva; a $15(17,85 \%)$ se trató de forma expectante; a $9(10,71 \%)$ se dió tratamiento médico con MTX y a $9(10,71 \%)$ se le administró tratamiento local combinado (citotóxico local y MTX parenteral).

9 pacientes reunieron los criterios de inclusión de EE no complicado con indicación quirúrgica de acuerdo a nuestras guías actuales de manejo de ginecología publicadas en el año 2011 del INMP a las que se les brindó tratamiento combinado con citotóxico local y MTX vía Intramuscular.

Las características de las pacientes se reportan en la Tabla 1.

La edad promedio de los pacientes intervenidos fue $28,0 \pm 5,0$ años, el tiempo quirúrgico promedio fue de
Tabla 1. Características clínicas de las pacientes con embarazo ectópico no complicado con indicación quirúrgica, en el servicio de ginecología del INMP.

\begin{tabular}{lc}
\hline Datos clínicos & $\bar{X} \pm$ D.S. (Mín. - Máx.) \\
\hline Edad. & $28 \pm 5(19-34)$ \\
$\begin{array}{l}\text { Valor de HCG cuantitativa } \\
\text { (mUl/ml). }\end{array}$ & $9500 \pm 3384(3886-14783)$ \\
Tiempo quirúrgico (en min.). & $12 \pm 5(5-24)$ \\
Síntomas. & \\
Dolor pélvico & $3(33,3 \%)$ \\
Dolor abdominal & $1(11,1 \%)$ \\
Dolor pélvico y sangrado & $5(55,6 \%)$ \\
vaginal escaso & \multicolumn{2}{c}{ Total } & $\mathbf{9 ( 1 0 0 , 0 \% )}$ \\
\hline \multicolumn{2}{c}{}
\end{tabular}

Tabla 2. Diagnóstico y tratamiento de las pacientes con embarazo ectópico no complicado con indicación quirúrgica, en el servicio de ginecología del INMP.

\begin{tabular}{lc}
\hline Diagnóstico ecográfico. & $\mathbf{N}(\%)$ \\
\hline Saco gestacional & $4(44,4 \%)$ \\
\hline Saco gest / LCN / LCF & $5(55,6 \%)$ \\
\hline Localización del ectópico. & \\
\hline En cicatriz de cesárea anterior & $1(11,1 \%)$ \\
\hline Cervical & $1(11,1 \%)$ \\
\hline Tubárico izquierdo & $5(55,5 \%)$ \\
\hline Tubárico derecho & $2(22,2 \%)$ \\
\hline Citotóx Local. & \\
\hline CLK & $2(22,2 \%)$ \\
\hline MTX Total & $7(77,8 \%)$ \\
\hline
\end{tabular}

$12,0 \pm 5,0$ minutos y respecto a los hallazgos de laboratorio, se observó que el valor medio del HCG fue $9500 \pm 3384$ $\mathrm{mUl} / \mathrm{ml}$. Entre los síntomas se observó dolor pélvico en 3 pacientes $(33,3 \%)$, dolor abdominal en un paciente $(11,1 \%)$ y dolor pélvico y sangrado vaginal en 5 pacientes $(55,6 \%)$.

Sobre los hallazgos ecográficos de los pacientes con embarazo ectópico, en 4 pacientes $(44,4 \%)$ se observó saco gestacional (SG) y en 5 pacientes $(55,6 \%)$ saco gestacional y medición del LCN (longitud corono-nalga). Y según la localización del embarazo ectópico, en cinco pacientes $(55,5 \%)$ se localizó tubárico izquierdo, 2 pacientes $(22,2 \%)$ tubárico derecho, un paciente $(11,1 \%)$ en la cicatriz de la cesárea anterior, un paciente $(11,1 \%)$ en la cervical.

Tabla 3. Datos de las 9 pacientes con manejo combinado de Metotrexate atendidas en el servicio de ginecología del INMP.

\begin{tabular}{|c|c|c|c|c|c|c|c|c|c|c|}
\hline Paciente & Edad & Paridad & $\begin{array}{c}\text { Edad } \\
\text { Gestac }\end{array}$ & Sintomas & $\begin{array}{c}\text { Nivel de } \\
\text { HCG }\end{array}$ & Dx Ecog. & $\begin{array}{c}\text { MTX } \\
\text { previo }\end{array}$ & $\begin{array}{l}\text { Localiz } \\
\text { Ectópico }\end{array}$ & $\begin{array}{c}\text { Citotóx } \\
\text { Local }\end{array}$ & $\begin{array}{c}\text { Tiempo } \\
\mathbf{Q x}\end{array}$ \\
\hline 1 & 28 & G1P0 & 7 sem & $\mathrm{DP}+\mathrm{SV}+$ & 9780 & SG & 1 doo IM & Tub Izq & MTX & $9 \mathrm{~min}$ \\
\hline 2 & 25 & G2P0010 & 7 sem & $\mathrm{DP}+\mathrm{SV}+$ & 10840 & $\begin{array}{c}\text { SG, LCN Icf } \\
124\end{array}$ & (x) & Tub Izq & MTX & $16 \mathrm{~min}$ \\
\hline 3 & 34 & G4P2012 & 7 sem & $\mathrm{DP}+\mathrm{SV}+$ & 6737 & SG & - & Tub Der & MTX & $11 \mathrm{~min}$ \\
\hline 4 & 29 & G1P0 & 6 sem & $\mathrm{DP}+$ & 7260 & SG, LCN Icf - & - & Tub Izq & MTX & $14 \min$ \\
\hline 5 & 22 & G3P0 & 6 sem & $\mathrm{DP}+\mathrm{SV}+$ & 11700 & $\begin{array}{c}\text { SG, LCN Icf } \\
128\end{array}$ & - & Tub Der & MTX & $12 \min$ \\
\hline 6 & 34 & G5P3013 & 7 sem & $\mathrm{DA}+$ & 3886 & SG & 1 doo IM & Cicatriz de CA & ClK & $10 \mathrm{~min}$ \\
\hline 7 & 19 & G1P0 & 5 sem & $\mathrm{DP}+\mathrm{SV}+$ & 7842 & $\begin{array}{c}\text { SG, LCN Icf } \\
105\end{array}$ & - & Tub Izq & MTX & $24 \min$ \\
\hline 8 & 30 & G4P2012 & 6 sem & DP & 12670 & SG & - & Cervical & CIK & $5 \min$ \\
\hline 9 & 31 & G3P0 & 10 sem & DP & 14783 & SG,LCN Icf - & - & Tub Izq & MTX & $10 \min$ \\
\hline
\end{tabular}


Entre los tratamientos recibido por los pacientes con embarazo ectópico, 2 (22,2\%) recibieron CLK y 7 (77,8\%) recibieron metotrexato (MTX).

Tabla 4. Tipo de tratamiento en pacientes con embarazo ectópico no complicado con indicación quirúrgica

\begin{tabular}{lc}
\hline Tipo tratamiento & $\mathbf{N ~ ( \% )}$ \\
\hline Quirúrgico & $51(60,7 \%)$ \\
Expectante & $15(17,9 \%)$ \\
Médico & $9(10,7 \%)$ \\
Local Metrotexate & $9(10,7 \%)$ \\
\hline Total & $\mathbf{8 4}(100 \%)$ \\
\hline
\end{tabular}

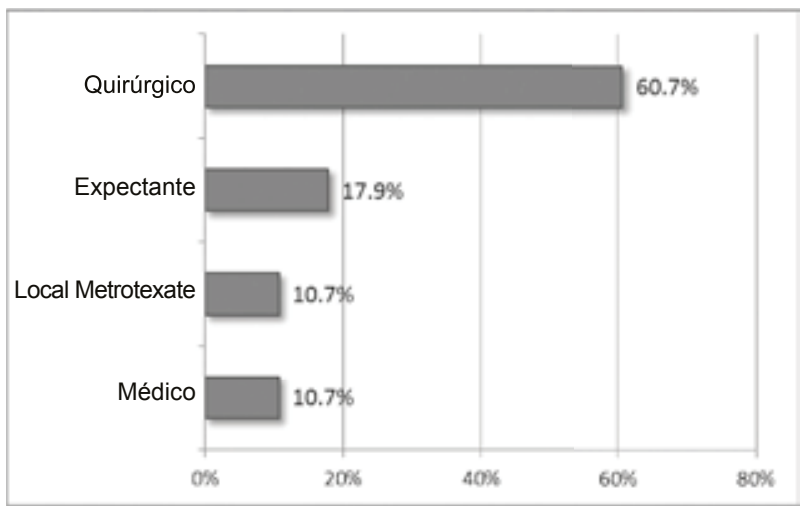

Gráfico 1. Tipo de tratamiento en pacientes con embarazo ectópico no complicado con indicación quirúrgica

Tabla 5. Tiempo de hospitalización según tipo de tratamiento en pacientes con embarazo ectópico no complicado con indicación quirúrgica

\begin{tabular}{lc}
\hline \multirow{3}{*}{ Tipo de tratamiento } & $\begin{array}{c}\text { Tiempo de } \\
\text { hospitalización (días) }\end{array}$ \\
\cline { 2 - 2 } & $\overline{\mathrm{X} \pm \mathrm{DS} \text { (Min-Max) }}$ \\
\hline Tratamiento expectante & $3,5 \pm 1,5(2-7)$ \\
Tratamiento médico & $6,8 \pm 2(4-9)$ \\
Tratamiento quirúrgico & $2,6 \pm 0,+0,9(1-6)$ \\
Tratamiento local combinado & $6,8 \pm 3,3(3-11)$ \\
\hline
\end{tabular}

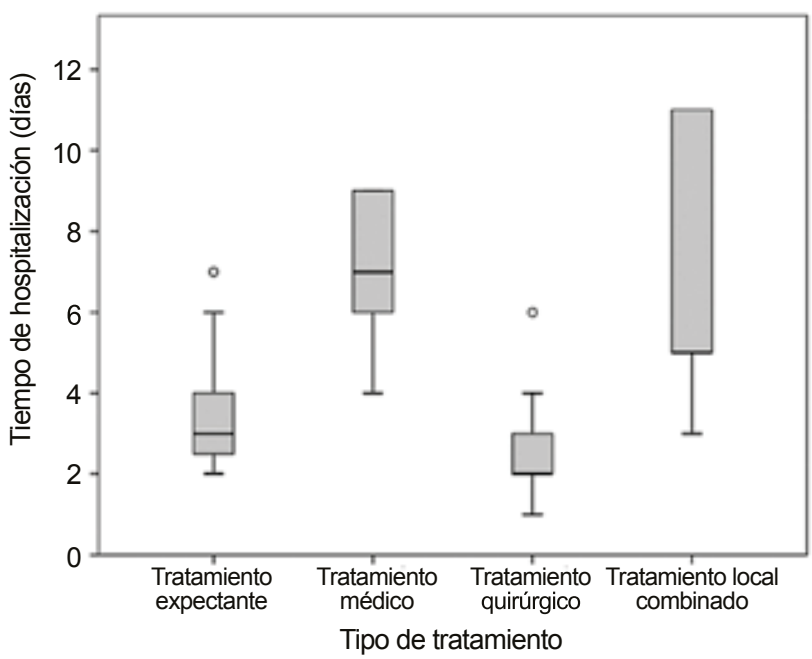

Gráfico 2. Comparación de Tiempo de hospitalización según tipo de tratamiento en pacientes con embarazo ectópico no complicado con indicación quirúrgica
Según el tipo de tratamiento se observa que el médico tiene un promedio de $6,8 \pm 2$ días, el tratamiento quirúrgico tiene una media de $2,6 \pm 0,+0,9$ días, el tratamiento local combinado duró la hospitalización una media de $6,8 \pm 3,3$ días y finalmente el tratamiento expectante tiene un promedio de $3,5 \pm 1,5$ días.

\section{DISCUSIÓN}

La cirugía es el tratamiento standard en el embarazo ectópico no complicado con indicación quirúrgica ${ }^{24}$. En general se realiza una salpinguectomía, ya que una cirugía conservadora además de estar asociado a sangrado intraoperatorio y postoperatorio entraña un riesgo de hasta $8 \%$ de ectópico residual ${ }^{25,26}$.

La tendencia actual de la cirugía es cada vez hacer intervenciones de mínima invasión como NOTES (Cirugía endoscópica transluminal a través de orificios naturales), técnica que utiliza como vía de abordaje orificios naturales como boca, uretra, ano, vagina, colon; actualmente todavía en desarrollo, pero considerada como segura ${ }^{27}$.

Se ha empleado también a la ecografía ya no sólo como evaluación y diagnóstico, si no como ayuda para realizar procedimientos que antes se hacían a ciegas (infiltración en articulaciones) o mediante intervención quirúrgica (aspiración folicular), es lo que se llama ahora ecografía intervencionista; muestra de ello es la gran contribución el sustituir la aspiración folicular por laparoscopia a aspiración folicular ecoguiada.

Nuestros resultados confirman la efectividad del esquema utilizado: Metrotexate Local intrasacular ecoguiado $(50 \mathrm{mg}$ ) y Metrotexate intramuscular dosis única $50 \mathrm{mg} / \mathrm{mt} 2 \mathrm{de}$ superficie. Sólo una de las nueve pacientes tratadas tuvo que ser intervenida quirúrgicamente por embarazo ectópico roto. Estos resultados son similares a reportes previos en quienes mostraron más fallas con tratamiento sistémico comparado con tratamiento local. No se reportó mayor efecto sistémico con el tratamiento combinado (local y sistémico) en los controles post procedimiento con perfiles de laboratorio tanto renal, hepático o hematológico. No se reportó ningún accidente en la intervención tanto por el operador, ni por el anestesiólogo.

Sólo el tratamiento local ha sido tan efectivo en pacientes con indicación médica, que Hafner $\mathrm{T}$ y col. Decidieron cambiar de usar metrotexate sistémico a usar metrotexate local a todas los casos de embarazo ectópico ${ }^{19}$. Los datos reportados en la literatura y nuestra experiencia sugieren la superioridad del tratamiento local, tanto en relación a la seguridad como a la efectividad.

El tratamiento del embarazo ectópico sigue en evolución y aún no está dicha la última palabra y está relacionada al avance de la medicina moderna y ésta no se detiene. Por lo que seguiremos solucionando problemas con el mejor perfil costo efectividad para el paciente. En ese 
sentido también evaluamos el tiempo del procedimiento siendo el tiempo promedio de 12.5 minutos en sala de operaciones. Tiempo que es mucho menos al reportado para solucionar el EE según un estudio clínico randomizado, prospectivo donde evalúan el tiempo operatorio para laparoscopía de 73 minutos vs. 88 minutos para el grupo de laparotomía ${ }^{28}$.

Las opciones de tratamiento para el embarazo ectópico no complicado con indicación quirúrgica estarán relacionadas a la aparición de nuevas técnicas de abordaje aún en desarrollo, de nuevos conocimientos en relación al impacto fisiopatológico que pueda generar la disminución de la vascularización al realizar una salpingectomía con las técnicas estándares y en relación a la paridad de la paciente y calidad de vida que se le vaya a brindar, teniendo en cuentas estudios que exponen la disminución de la reserva ovárica o adelanto de la menopausia en pacientes con salpinguectomía.

En medicina reproductiva se ha tomado atención a artículos publicados como el daño en el flujo vascular del ovario así como la reducción del conteo de folículos antrales después de una salpinguectomía laparoscópica por embarazo ectópico ${ }^{29}$. Sin embargo otro estudio de Fertilización In Vitro posterior a una salpinguectomía por embarazo ectópico no encontró diferencias significativas en dosis o duración de gonadotropinas o niveles de estradiol. Además no encontró diferencias en el número de ovocitos aspirados o calidad embrionaria de los ciclos ${ }^{30}$.

Un estudio comparó la oclusión tubárica proximal vs salpinguectomía para tratar el efecto adverso del hidrosalpinx en los ciclos de Fertilización In Vitro y reportó que los niveles de FSH se incrementaron desde su línea de base después de la salpinguectomía, pero no para las pacientes a las que se les sometió a oclusión tubárica proximal ${ }^{31}$.

Un estudio controlado, rabdomizado, encontró que la esterilización tubárica mediante cauterización bipolar tenía un efecto adverso sobre el conteo de folículos antrales y el volumen del ovario, mientras que este efecto no se evidenció cuando la ligadura se realizó con clips mecánicos ${ }^{32}$.

En medicina reproductiva tratando de no tocar la circulación útero ovárica con las posibles consecuencias negativas para el ovario se han propuesto nuevos opciones de tratamiento para evitar la salpinguectomía; así por ejemplo el tratamiento estándar para el hidrosalpinx es la salpinguectomía, sin embargo están apareciendo reportes de series de casos de oclusión tubárica proximal intraluminal mediante la inserción histeroscópica de Essure (dispositivo originalmente creado para esterilización tubárica) ${ }^{33,34}$. Así mismo se ha reportado de aspiración del hidrosalpinx bajo guía ecográfica en el momento de la aspiración ovocitaria para evitar la cirugía ${ }^{35,36}$.
Hace falta entonces estudios prospectivos metodológicamente bien diseñados para evaluar el impacto de la salpinguectomía en la circulación ovárica y por tanto en la reserva ovárica y/o adelanto de la menopausia de la mujer. Mientras siga la controversia empezaremos por ser los más conservadores posibles, más aún con la aparición de técnicas mini invasivas con igual o mejor perfil de éxito y confort para el paciente.

Por el momento las ventajas del tratamiento con metrotexate ecoguiado intrasacular e intramuscular a pacientes con EE no complicado que necesitan una cirugía son evitar una laparotomía o una laparoscopía, menor tiempo de intervención, por lo tanto menor exposición anestésica, recuperación inmediata, parece ser costo efectivo. Las desventajas serían y éstas podrían ser superadas en el tiempo como personal médico con entrenamiento en ecografía intervencionista, equipo e insumos en sala de operaciones para el procedimiento, control y seguimiento largo post procedimiento de las pacientes intervenidas.

Quedaría también realizar trabajos prospectivos longitudinales evaluando variables bioquímicas como Hormona Antimulleriana, tanto a pacientes que se realizarán una salpinguectomía como aquellas que se someterán sólo a tratamiento combinado con MTX con una muestra adecuada que permita tener conclusiones o evaluar controles seriados de reserva ovárica en grupos de pacientes comparables en edad; y en el tiempo evaluar valores basales de reserva ovárica, ya que el estado de embarazo altera los valores bioquímicos convencionales de reserva ovárica.

\section{REFERENCIAS BIBLIOGRÁFICAS}

1. Oron G, Tulandi T. A pragmatic and evidence-based management of ectopic pregnancy. J Minim Invasive Gynecol 2013;20(4):446-54.

2. Chang J, Elam-Evans LD, Berg CJ, Herndon J, Flowers L, Seed KA, et al. Pregnancy related mortality surveillance-United States, 1991-1999. MMWR Surveill Summ 2003;52:1-9.

3. Ankum WM, Mol BW, Van der Veen F, Bossuyt PM. Risk factors for ectopic pregnancy: a meta-analysis. Fertil Steril 1996;65:1093-9.

4. American College of Obstetrician and Gynecologist, Medical management of ectopic pregnancy. ACOG Practice Bulletin 94. 2008.

5. Varma R, Gupta J. Tubal ectopic pregnancy. Clin Evid Handbook. June 2013:521-523.

6. Autry A. Medical treatment of ectopic pregnancy, Is There Something New? Editorial American Colllege of Obstetrics \& Gynecology vol. 122. N4: 733-734 October 2013

7. Fernandez H, Capmas P, Lucot JP, Resch B, Panel P, Bouyer J. Fertility after ectopic pregnancy: the DEMETER randomized trial. Human Reproduction. 2013;28(5):1247-1253.

8. Mol BW, Hajenius PJ, Engelsbel S, Ankum WM, van der Veen F, Hemrika DJ, Bossuyt PM. Serum human chorionic gonadotropin measurement in the diagnosis of ectopic pregnancy when transvaginal sonography is inconclusive. Fertil Steril 1998;70:972-981. 
9. Van Mello NM y col. Methotrexate or expectant management in women with an ectopic pregnancy or pregnancy of unknown location and low serum hCG concentrations? A randomized comparison. Hum. Reprod. Vol 28, №1:60-67, 2013.

10. Barnhart $K$, van Mello NM, Bourne $T$, Kirk E, Van CB, Bottomley C, Chung K, Condous G, Goldstein S, Hajenius PJ et al. Pregnancy of unknown location: a consensus statement of nomenclature, definitions, and outcome. Fertil Steril 2011;95:857-866

11. Hajenius PJ, Mol F, Mol BW, Bossuyt PM, Ankum WM, van der Veen F. Interventions for tubal ectopic pregnancy. Cochrane Database Syst Rev 2007:CD000324.

12. Banerjee S, Aslam N, Woelfer B, Lawrence A, Elson J, Jurkovic $D$. Expectant management of early pregnancies of unknown location: a prospective evaluation of methods to predict spontaneous resolution of pregnancy. $\mathrm{Br} \mathrm{J}$ Obstet Gynaecol 2001;108:158-163.

13. Patil M. Ectopic pregnancy after infertility treatment. [Journal Article]. J Hum Reprod Sci 2012 May; 5(2):154-65.

14. Sowter MC, Farquhar CM, Petrie KJ, Gudex G. A randomised trial comparing single dose systemic methotrexate and laparoscopic surgery for the treatment of unruptured tubal pregnancy. Br J Obstet Gynecol 2001;108:192-203.

15. Royal College of Obstetricians and Gynaecologists. The management of tubal pregnancies. In: 'Green top' Guideline No.21. London: Royal College of Obstetricians and Gynaecologists; 1999.

16. Ozmen B, Diedrich K, Al-Hasani S. Hydrosalpinx and IVF: Assessment of treatments implemented prior to IVF. Reprod Biomed Online 2007;14:235-41.

17. Lass A, Ellenbogen A, Croucher C, Trew G, Margara R, Becattini $C$, et al. Effect of salpingectomy on ovarian response to superovulation in an in vitro fertilization-embryo transfer program. Fertil Steril 1998;70:1035-8.

18. Meng $X H$, Zhu YM. Effect of salpingectomy on ovarian function. Zhejiang Da Xue Xue Bao Yi Xue Ban 2006;35:5559.

19. Hafner T, Aslam N, Ross JA, Zosmer N, Jurkovic D. The effectiveness of non-surgical management of early interstitial pregnancy: a report of ten cases and review of the literature Ultrasound Obstet Gynecol 1999;13:131-136.

20. Monteagudo A, Minior VK, Stephenson C, Monda S Timor-Tritsch IE. Non-surgical management of live ectopic pregnancy with ultrasound-guided local injection: a case series Ultrasound Obstet Gynecol 2005; 25: 282-288.

21. Seow KM, Wang PH, Huang LW, Hwang JL. Transvaginal sono-guided aspiration of gestational sac concurrent with a local methotrexate injection for the treatment of unruptured cesarean scar pregnancy. Arch Gynecol Obstet. 2013;2:361-6

22. Pagidas K, Frishman GN. Nonsurgical management of primary ovarian pregnancy with transvaginal ultrasound- guided local administration of methotrexate. J Minim Invasive Gynecol. 2013;20(2):252-4.

23. Dai $Q$, Wang LL, Shao $X H$, Wang $S M$, Dong $X Q$. Clinical effectiveness of multiple-drug injection treatment in unruptured ectopic pregnancies: a retrospective study. J Ultrasound Med. 2012;31(10):1627-34

24. Johnston J. Ectopic pregnancy. Clinic Obstet and Gynecol 2007; $50: 89-99$

25. Sowter MC, Farquhar CM. Ectopic pregnancy: an update. Curr Opin Obstet Gynecol. 2004;16:289-293.

26. Hajenius PJ, Mol BW, Ankum WM, et al. Clearance curves of serum human chorionic gonadotrophin for the diagnosis of persistent trophoblast. Hum Reprod. 1995;10:683-687.

27. Thele F, Zygmunt M, Glitsch A, Heidecke CD, Schreiber A. How do gynecologists feel about transvaginal NOTES surgery? Endoscopy. 2008;40(7):576-80.

28. Lundorff $P$, Thorburn J, Hahlin M, Källfelt B, Lindblom B. Laparoscopic surgery in ectopic pregnancy: $A$ randomized trial versus laparotomy. Acta Obstet \& Gynecol Scandinavica. 1991;70(4-5):343-348.

29. Chan CC, Ng EH, Li CF, Ho PC. Impaired ovarian blood flow and reduced antral follicle count following laparoscopic salpingectomy for ectopic pregnancy. Hum Reprod 2003;18:2175-80

30. Dar P, Sachs GS, Strassburger D, Bukovsky I, Arieli S. Ovarian function before and after salpingectomy in artificial reproductive technology patients. Hum Reprod 2000;15:142-4.

31. Nakagawa $K$, Ohgi $S$, Horikawa $T$, Irahara $M$, Saito $H$. Laparoscopic proximal tubal division can reserve for infertility patients with hydrosalpinges. J Obstet Gynaecol Res 2008;34:1037-42.

32. Goynumer G, Kayabasoglu F, Aydogdu S, Wetherilt L. The effect of tubal sterilization through electrocoagulation on the ovarian reserve. Contraception 2009;80:90-4

33. Mijatovic V, Veersema S, Emanuel MH, Schats R, Hompes PG. Essure hysteroscopic tubal occlusion device for the treatment of hydrosalpynx prior to in vitro fertilization-embryo transfer in patients with a contraindication for laparoscopy. Fertil Steril 2010;93:1338-42

34. Darwish AM, EI Saman AM. Is there a role for hysteroscopic tubal occlusion of functionless hydrosalpinges prior to IVF/ICSI in moder practice? Acta Obstet Gynecol Scand 2007;86:1484-9.

35. Sowter MC, Akande VA, Williams JA, Hull MG. Is the outcome of in-vitro fertilization and embryo transfer treatment improve by spontaneous or surgical drainage of a hidrosalpinx? Hum Reprod 1997;12:2147-50.

36. Van Voorhis BJ, Sdarks AE, Syrop CH, Stovall DW. Ultrasound-guided aspiration of hydrosalpinges is associated with improved pregnancy and implantation rates after in-vitro fertilization cycles. Hum Reprod 1998;13:736-9. 